\title{
Olfactory bulb volume and olfactory function in patients with multiple sclerosis*
}

\author{
Oender Goektas ${ }^{1}$, Felix Schmidt ${ }^{2}$, Georg Bohner ${ }^{3}$, Katharina Erb ${ }^{3}$, Lutz Lüde- \\ mann $^{3}$, Bettina Dahlslett ${ }^{1}$, Lutz Harms ${ }^{2}$, Franca Fleiner ${ }^{1}$ \\ 1 Department of Otolaryngology-Head and Neck Surgery, University of Berlin, Charité Campus Mitte, Smell \\ and Taste Consultation Service, Berlin, Germany \\ 2 Department of Neurology, Consultation Service for multiple sclerosis, University of Berlin, Charité Campus \\ Mitte, Berlin, Germany \\ 3 Department of Radiology, University of Berlin, Charité Campus Mitte, Berlin, Germany
}

SUMMARY

\section{INTRODUCTION}

Multiple sclerosis, a very complex poly-symptomatic disease, is one of the great, unsolved mysteries in modern medicine. It strikes young adults, runs a chronic, unpredictable course, and is eventually disabling for many patients. Central mechanisms are inflammation, demyelination, gliosis and neuro-degeneration in the brain and spinal chord ${ }^{(1)}$. Multiple sclerosis usually begins as a relapsing-remitting disease, but in most patients a steadily progressive neurological disorder ultimately ensues. The relapsing phase is often mediated by clinical exacerbations and focal bursts of inflammation in the white matter of the brain and the spinal chord, whereas axonal and neuronal loss predominates during the progressive phase ${ }^{(2)}$. The lesions are predominantly situated in the periventricular white matter, the corpus callosum, infratentorialy in the pons, and in the cerebellum ${ }^{(3)}$. The symptoms differ according to the affected region in the central nervous system. Typical symptoms are sensory disturbances, Lhermitte's sign, diplopia, unilateral optic neuritis, limb weakness, gait ataxia, and neurogenic bladder and bowel symptoms ${ }^{(4)}$. Although decreased ability to smell is among the first signs of Alzheimer's disease and idiopathic Parkinson's disease ${ }^{(5,6)}$, olfactory dysfunction is absent or occurs at much lower frequency in most other neurodegenerative disorders. The reason for this early manifestation is still unclear, suggesting that in the case of Parkinson's disease there is a $100 \%$ increase of dopaminergic cells primarily in the $\mathrm{OB}{ }^{(7)}$. Therefore, before the substantia nigra is affected, the OF becomes the first deficit in these patients. In the case of MS, considerable controversy exists concerning the presence of olfactory dysfunction. Trials investigating the OF report that MS patients perform worse than healthy control subjects with $38.5 \%{ }^{(8)}, 15 \%{ }^{(9)}$ and $22.5 \%{ }^{(10)}$ of the patients with olfactory dysfunction. There is proof of a clear inverse correlation between the number of plaques in the olfactory cortex and the olfactory function in patients with MS ${ }^{(8,11)}$. It remains unclear whether olfactory disturbances occur as an initial symptom of MS ${ }^{(12)}$. Due to various applicable tests investigating the OF, such as the UPSIT (University of Pennsylvania Smell Identification Test), the CC-SIT (12 items Cross Cultural Smell Identification Test) and OEPs (olfactory evoked potentials), it is difficult to make a fair comparison of the patients OF from 
different studies. Our study focuses on the affect of MS on the OB and the loss of smell of patients with MS. Results of magnetic resonance imaging (MRI) are compared with the OF and neurological and psychological parameters to provide an improved insight into the disease pattern. For the first time, MRI-results about the effect of MS on the OB are evaluated separately. High resolving sequences, contrast agent and a constructive-interference-in-steady-state (CISS) were applied to demonstrate the olfactory bulb accurately.

\section{MATERIAL AND METHODS}

\section{Patients}

Thirty-six patients with the diagnosis of multiple sclerosis (25 women, 11 men, mean age $\pm \mathrm{SD}, 41.5 \pm 12.2$ years, duration of disease $6.2 \pm 7.8$ years) were examined in the Department of Otorhinolaryngology - Head and Neck Surgery. The patients were included and excluded by the examination of a neurologist, an otorhinolaryngologist, and two special questionnaires. Included were patients with the diagnosis of multiple sclerosis according to the revised McDonald criteria (13).

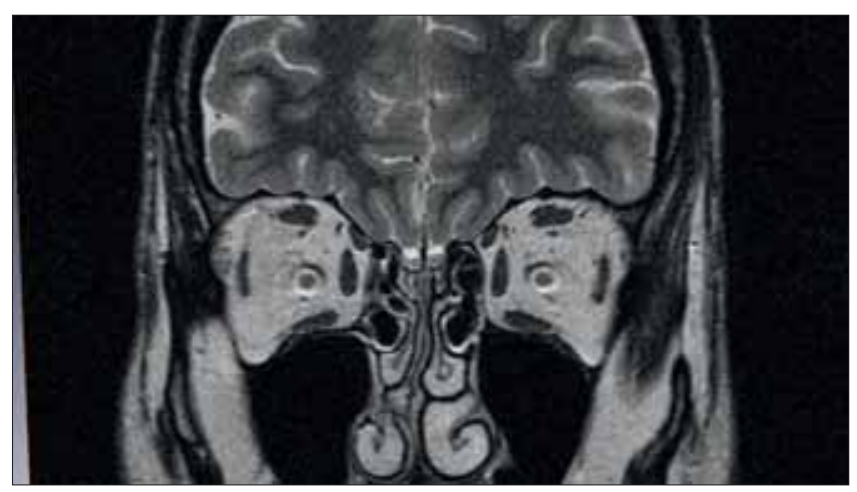

Figure 1. Olfactory bulb $(\mathrm{OB})$ in coronary view.

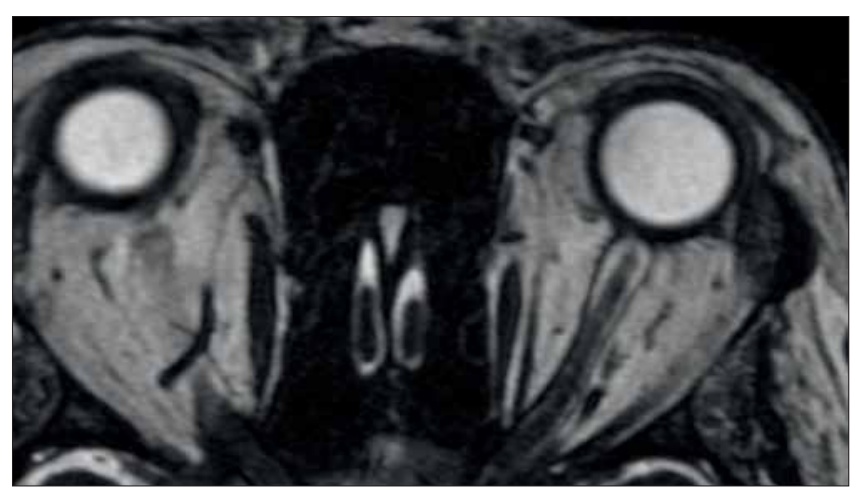

Figure 2. Olfactory bulb in axial view.

The OF of a healthy control group matching in age, sex and smoking habits was tested.

Ethical approval and trial registration was obtained by the medical ethics committee of Charité, University of Berlin. Written informed consent was approved by all participants. Exclusion criteria were pregnancy or lactation, age below 18 or above 65 years and exclusion criteria for participation at magnetic resonance imaging (severe cardiac disease, a pacemaker or other metal implants, recent stroke, surgery, kidney dysfunction, allergy to contrast agent, severe claustrophobia). Patients received a detailed ENT-examination including nasal endoscopy to evaluate the anatomy of the nasal cavities and especially the olfactory cleft, thereby excluding those patients whose sense of smell could possibly be affected by anatomical abnormalities. Patients with obstruction of the olfactory cleft by nasal polyps were not included in the study. Patients with other reasons affecting olfaction (post-traumatic olfactory dysfunction, sinonasal disease, the existence or therapy of a malignant tumour via radiotherapy and chemotherapy, post-upper respiratory tract infection (post-URTI), allergy, diabetes, hypertension, certain pharmaceuticals, Parkinson's disease, Alzheimer's disease, depression, diminution of smell related to age) were excluded. A Mini-Mental State Examination (MMSE) of at least 24 points was required to exclude cognitive dysfunction and Alzheimer's disease ${ }^{(14)}$. Physical problems of the patients that could interfere with interpretation of the questionnaire were considered during test evaluation. To exclude depression, a Becks Depression Inventory test (BDI) with less than 15 points was required. The BDI, revised in 1978, is a 21-question multiple-choice self-report inventory. It is one of the most widely used instruments for measuring the severity of depression. It is composed of items relating to symptoms of depression such as hopelessness and irritability, cognitions such as guilt or feelings of being punished, as well as physical symptoms such as fatigue, weight loss, and lack of interest in sex ${ }^{(15)}$. In patients with physical illness such as MS the BDI's reliance on physical symptoms such as fatigue may artificially inflate scores due to symptoms of the illness, rather than solely of depression. MS patients often have a higher level of depression than healthy control subjects. The test is scored differently for the general population and for individuals who have been clinically diagnosed with depression. The BDI score of 15 or higher was determined by a psychologist of Charité University in Berlin to exclude patients. Patients with scores higher than 15 were examined again by a psychologist who evaluated the Hamilton Rating Scale for Depression and the Hamilton Anxiety Rating Scale. An Expanded Disability Status Scale (EDSS) score of less than 8 ensured the ability of the patient to accomplish the tests and it demonstrated the disability status of the patient ${ }^{(16)}$. The EDSS score quantifies disability in 8 Functional Systems and allows neurologists to assign a Functional System Score in each of these. The Functional systems are: pyramidal, cerebellar, brainstem, sensory, bowel and bladder, visual, cerebral and other systems. A score between $0.0-10.0$ is assigned ( 0 : normal neurological examination, 1: no disability and a minimal sign on one functional system, 10: death due to MS). Psychophysical testing of the OF was performed using the tripartite TDI score (threshold-discrimination-identification) ${ }^{(17,18)}$. Thin section MRI of the brain with gadolinium enhancement was performed on the same day as the other testing. Eligible participants were continuously recruited from the Special Consulting Service for multiple sclerosis at the Neurology Clinic of Charite University 


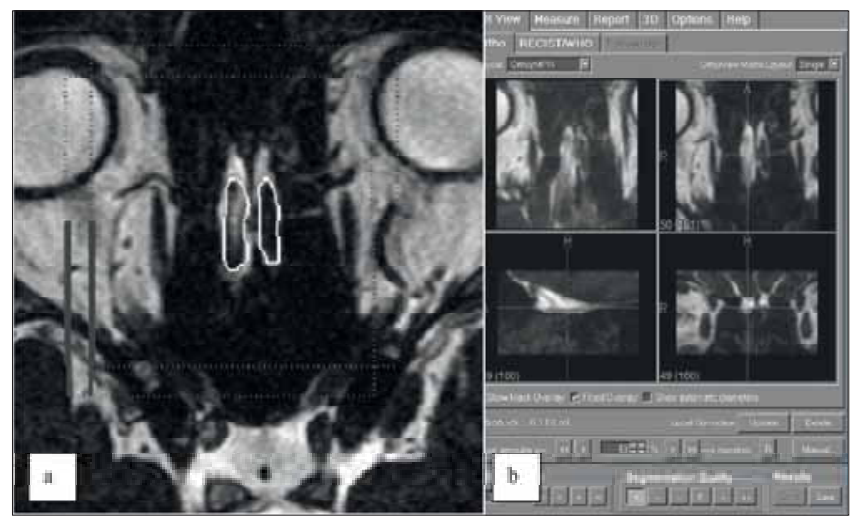

Figure 3. Manual segmentation (a) of the volume of the $\mathrm{OB}$ with MeVis OncoTreat V 1.6 with reformatted view (b) in axial, sagittal and coronal slices.

in Berlin from January until December 2009. Healthy control subjects matching in age and sex were mainly derived from healthy members of the hospital staff and family members of hospital patients during the same period. All participants underwent the testing procedures and clinical examination at the Special Consulting Service for Olfactory Disorders at the ENT-Department of the Charité University.

\section{Olfactory testing}

The TDI score measures the orthonasal OF and is recommended by the Working Group on Olfaction and Gustation of the German Society for Otorhinolaryngology, Head and Neck Surgery. The TDI score is a reliable ( $\mathrm{r}=$ 0.72 ) and validated test tool for testing $\mathrm{OF}^{(19)}$. It is a tripartite instrument measuring threshold, discrimination and identification with each of 16 odoriferous markers ('Sniffin Sticks' test battery) ${ }^{(17,18)}$. Participants are partly blinded and have to make a choice ('forced choice') in this procedure to identify their OF. The cap of the pen-like devices is removed and the pen's tip is positioned approximately $2 \mathrm{~cm}$ in front of both nostrils for a time period of 3s. In general a score of 16 or less indicates functional anosmia, a score up to 30 hyposmia, and above 30.5 normosmia.

\section{Magnetic Resonance Imaging (MRI)}

All patients had a full brain MRI using Magnetom Symphony Vision, Siemens Medical solutions 1.5-T signal scanner. Contrast agent allowed a detailed assessment of MS plaques. In a second MRI session, the protocol comprised slightly modified very fine T2-turbo-spin-echo sequences and a heavily T2-weighted 3-dimensional CISS sequence (constructive interference in steady state) with $0.5 \mathrm{~mm}$ slice thickness to visualize the $\mathrm{OB}$ and olfactory cortex (Figure 1, Figure 2). Volumetric measurement of the right and left $\mathrm{OB}$ was performed by manual segmentation as demonstrated in Figure 3. The measuring of the volume of the OB was done by two experienced neuroradiologists without knowledge of the olfactory test scores. The volume of the $\mathrm{OB}$ was measured to detect an atrophy of the $\mathrm{OB}$ and to correlate the volumes of the OBs with the OF.

\section{Statistical analysis}

Statistical analysis was performed with SPSS (17.0, Chicago, USA). The Mann-Whitney U test was used for independent samples comparing several groups. For variables according to a Gaussian distribution, the t-test was used. The correlation was assessed by a bivariate regression analysis. A difference was considered significant at a $\mathrm{p}$ value of $<0.05$.

\section{RESULTS}

Forty-three MS patients were recruited initially. Seven had to be excluded for the following reason: no confirmed diagnosis of MS. Twenty-five patients were diagnosed with 'relapsingremitting MS', five with 'primary chronic progressive MS' and four with 'secondary chronic progressive MS'. For two patients the MS form was not already determined. The volume of the OB within all MS patients was $129.1 \pm 41.5 \mathrm{~mm}^{3}$ with a range of $55.4-217.2 \mathrm{~mm}^{3}$. The volume of the left OB was 65.7 $\pm 22.6 \mathrm{~mm}^{3}$, of the right OB $63.4 \pm 21.7 \mathrm{~mm}^{3}$. The mean EDSSScore of the 36 patients was $3.3 \pm 2.1$, the mean MMSE-score was $28.9 \pm 1.1$, the mean BDI-score was $7.3 \pm 6.4$. Seventeen of the 36 patients $(47.2 \%)$ declared to smoke. Fourteen of the 36 patients $(38.9 \%)$ had recently taken cortisone. There was no significant correlation between $\mathrm{OB}$ volume and TDI score $(\mathrm{r}=0.142, \mathrm{p}=0.415)$ but a significant correlation between the volume of the OB and the MMSE score $(r=-0.420, p<0.05)$. The correlation between volume of the OB and the EDSS score was $r=0.244$, the BDI score $(r=0.283)$, duration of disease $(r$ $=0.246)$ and the latest relapsing phase $(r=-0.437)$.

The TDI within all MS patients was $31.5 \pm 4.0 .16$ of 36 patients $(44.4 \%)$ displayed hyposmia. None of the patients displayed anosmia. Twenty of the 36 patients were normosmic. Among the age, sex and smoking habits matching healthy control group the TDI was $32.9 \pm 4.0 .8 .3 \%$ of the participants displayed hyposmia, none displayed anosmia and $91.7 \%$ displayed normosmia. The Threshold subtest in MS patients $(\mathrm{n}=36)$ was $6.3 \pm 1.8$, for the healthy control group this was $6.2 \pm 1.8$. In the Discrimination subtest the MS patients

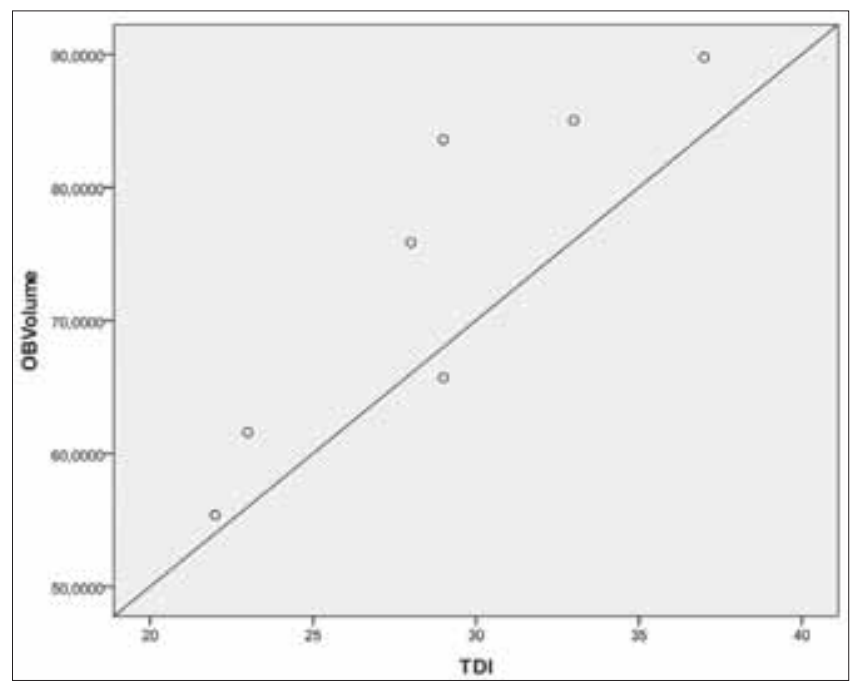

Figure 4. MS patients scored significantly poorer $(\mathrm{p}<0.05)$ than the control group in the Identification subtest. 
had $12.4 \pm 2.5$, the control group had $12.5 \pm 2.3$. In the Identification subtest, the MS patients scored significantly poorer ( $\mathrm{p}<0.05$ ) with $12.8 \pm 1.7$ than the control group 14.1 \pm 2.1 , demonstrated in Figure 4. The TDI score correlated significantly with the EDSS score $(r=-0.459, p=0.006)$. There was a significant correlation between the Identification subtest (I) and EDSS score $(r=-0.569, p=0.000)$, the latest relapsing phase $(r=0.927, p=0.000)$ and duration of disease $(r=-0.372$, $p=0.026)$. The Threshold subtest and Discrimination subtest showed no significant correlation to the other parameters.

Nine of all 36 MS patients had a volume of the OB below $100 \mathrm{~mm}^{3}$ with $74.0 \pm 12.8 \mathrm{~mm}^{3}$. Recent studies reported a volume of the $\mathrm{OB}<100 \mathrm{~mm}^{3}$ as cut-off for hyposmia (20). The TDI score of these patients was $29.7 \pm 4.7 \mathrm{~mm}^{3}$. Six of the 9 patients displayed hyposmia (66.7\%), 3 patients $(33.3 \%)$ displayed normosmia. When examining the findings of the 9 patients there was a significant correlation found between the volume of the OB and the TDI score $(r=0.825, p<0.01)$ demonstrated in Figure 5. There was a significant correlation between the volume of the $\mathrm{OB}$ with the Threshold subtest $(\mathrm{r}=$ $0.702, p<0.05)$ and Identification subtest $(r=0.773, p<0.05)$, but not with the Discrimination subtest $(\mathrm{r}=0.553)$. The OB volume correlated stronger with the EDSS score $(r=-0.586)$.

\section{DISCUSSION}

The sense of smell is of high importance for the perception of our environment in everyday life. It provides a basic warning function and influences the quality of life, for example the enjoyment of food or the selection of a partner. The OF has a great effect on the psyche as many connections between the olfactory cortex and different parts of the central nervous system exist. Olfactory dysfunction is often part of depression (21). The detection and treatment of olfactory dysfunction is of high importance and should not be underestimated. Olfactory dysfunction is often a first symptom of a neurodegenerative disease such as Parkinson or Alzheimer disease and can be a helpful sign for early clinical diagnostics. MRI of the OB

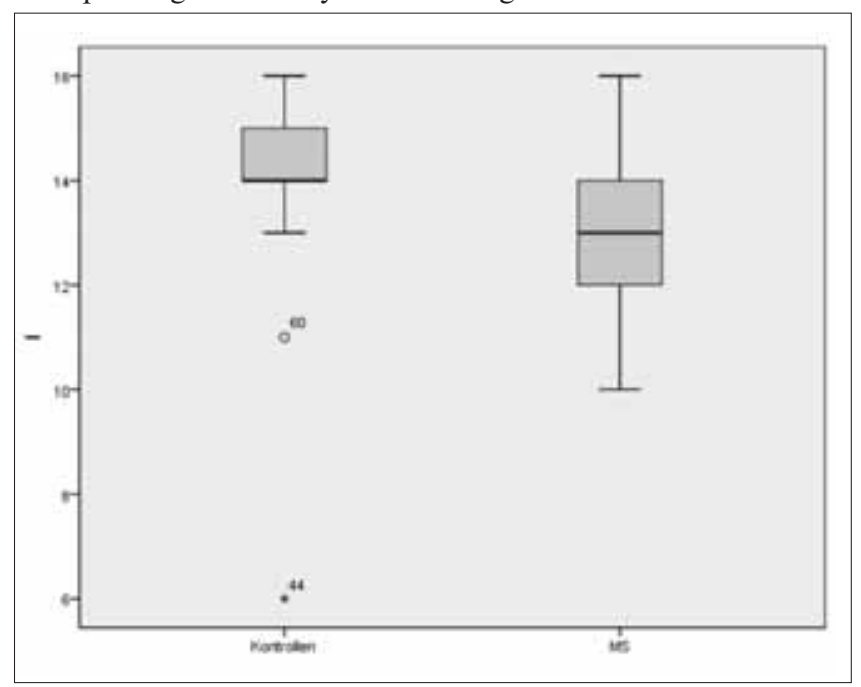

Figure 5. Significant correlation between the volume of the $\mathrm{OB}$ and the TDI score $(r=0.825, p<0.01)$ in patients with hyposmia. appears to be of interest in detection of olfactory dysfunction. It seems likely that changes of the OB provide a measure of the prognosis of $\mathrm{OF}^{(22,23)}$. Many studies demonstrated that $\mathrm{OB}$ volumes related to the OF both in normal and pathological conditions (post-traumatic olfactory loss, chronic rhinosinusitis). It has been observed that the $\mathrm{OB}$ volume changes with the degree of olfactory dysfunction. Results from studies suggest that $\mathrm{OB}$ volume is sensitive even to little changes in the olfactory system, the volume of the human OB reflecting individual changes in olfactory status ${ }^{(23)}$. Goektas et al. reported a significant correlation between $O B$ volumes of 24 patients with olfactory dysfunction (postviral, posttraumatic, idiopathic) with objective olfactometry ${ }^{(24)}$. In a recent study, ROC-analysis (sensitivity 0.95 , specificity 0.9 ) determined an $\mathrm{OB}$ volume of $100 \mathrm{~mm}^{3}$ as cut-off for olfactory hyposmia. This cut-off gives an indication that is debatable (20). MRI of the OB in healthy, normosmic subjects suggests that the volume of the $\mathrm{OB}$ is highly variable, especially in relation to ageing ${ }^{25)}$. The reason for the high plasticity is due to the continuing synaptogenesis in the $\mathrm{OB}$, which remains highly plastic throughout adult life. Bulbar neurogenesis relates to the activity level of sensory input from the olfactory epithelium (26) leading to a reduction in OB size or an improvement of sensory abilities ${ }^{(27)}$. Considering neurologic and psychiatric disorders, OB volumes of patients with idiopathic Parkinson's disease were not decreased compared to controls and did not correlate with OF expressed as TDI score. These data support the idea that olfactory loss in Parkinson's disease results from central-nervous changes rather than from damage to the olfactory epithelium and $\mathrm{OB}{ }^{(28)}$. In Alzheimer's disease and schizophrenia, OB volumes were decreased compared to controls ${ }^{(23)}$. In the case of MS, the reason for olfactory dysfunction remains unclear. Different studies demonstrated that the OF of a sizable proportion of MS patients with olfactory dysfunction correlates with plaque activity within olfactory-related central brain regions ${ }^{(8,11)}$. The OB of MS patients has not been examined separately yet. Therefore, the OB volume of patients with MS was measured and compared with the OF function of these patients in our study. It should be further investigated with a larger number of MS patients. Our study could not report a significant correlation between the OB volume of all MS patients and the TDI score. The OB volume of $9 \mathrm{MS}$ patients displaying a volume below 100 $\mathrm{mm}^{3}$ correlated significantly with the OF expressed as TDI score. Six of them displayed hyposmia. It confirms the results of recent studies that $\mathrm{OB}$ volume changes with the degree of olfactory dysfunction ${ }^{(23)}$.

Many MS patients participating in our study reported individual changes in their OF with amelioration and aggravation of the OF during disease progression. We will further investigate this aspect in a longitudinal study.

Forty-four percent of the MS patients displayed a clinical hyposmia evaluated by the TDI score. It confirms the existing percentage of olfactory dysfunction in MS patients presented in the published literature and is even higher. Different 
studies showed olfactory dysfunction in MS patients in 15 $38.5 \%$ of the cases. This could be due to the TDI test being more sensitive for patients with MS, measuring Threshold, Discrimination and Identification. We considered the age of the patients for a more precise evaluation of the test scores. The EDSS score quantifying the disability status changes during disease progression depending on remitting or relapsing phase with bursts of inflammation. The significant negative correlation between TDI and EDSS score and the high percentage of hyposmic MS patients suggests that the TDI score is sensitive for detecting olfactory changes in patients with MS and gives an accurate insight into the disease activity ('wax and wane theory').

Olfactory testing could help to indicate a relapsing phase early in order to improve the anti-inflammatory treatment. This aspect should be further investigated.

The tripartite TDI test was applied the first time for measuring OF in MS patients. It seems to be superior to other psychophysical testing procedures measuring orthonasal OF in MS patients. In different studies, OF in MS patients was tested using UPSIT and CC-SIT tests ${ }^{(8-11)}$. These smell tests check the identification of odours but not the discrimination and threshold. No correlation between these olfactory tests and EDSS score was found, a lower percentage of olfactory dysfunction was detected. In the Identification subtest, the MS patients scored significantly lower with 1.3 points than the healthy control group. Figure 4 demonstrates that the I-score of MS patients is equally distributed around their mean of 12.8 point. In the control group, just two patients scored below the mean of 14.1, the Identification score is right shifted. The Identification subtest of MS patients correlated highly significantly with the EDSS score, latest relapsing phase and duration of disease. These parameters are very typical of MS and important for clinical evaluation. These findings suggest the Identification subtest with its 16 different odours being very sensitive for measuring OF in MS patients. It is a very quick, efficient and inexpensive method for psychophysical testing of $\mathrm{OF}$.

\section{CONCLUSION}

A considerable percentage of MS patients displayed hyposmia. OB volumes may provide valuable information about MS patients with olfactory dysfunction. Volumetric measurement results of the $\mathrm{OB}$ of patients with a decreased $\mathrm{OB}$ volume correlated strongly with their OF. The TDI test seems to be superior to the UPSIT and CC-SIT test that were applied in recent studies for measuring OF in MS patients. The Identification subtest could be used as a quick screening method for olfactory dysfunction in MS patients. The findings might contribute to therapeutic decision making and provide prognostic information, but repeated examinations in longitudinal studies with a larger number of MS patients are still required.

\section{CONFLICT OF INTEREST}

We confirm that this work is not involved into financial or other relationships, which may lead to a conflict of interest.

\section{REFERENCES}

1. Brück W, Neubert K, Berger T, Weber JR. Clinical, radiological, immunological and pathological findings in inflammatory CNS demyelination - possible markers for an antibody-mediated process. Mult Scler. 2001, 7: 173-177.

2. Confavreux C, Vukusic S, Moreau T, Adeleine P. Relapses and progression of disability in multiple sclerosis. N Engl J Med. 2000, 343: 1430-1438.

3. Brex PA, Ciccarelli O, O'Riordan JI, Sailer M, Thompson AJ, Miller DH. A longitudinal study of abnormalities on MRI and disability from multiple sclerosis. N Engl J Med. 2002, 346: 158164.

4. Schmidt RM, Neumann V, Kissig B, Kuppe G, Ludewig R. Darstellung ausgewählter Untersuchungen zum Krankheitsbild der Multiplen Sklerose. Nervenarzt. 1987, 58: 403-412.

5. Ponsen MM, Stoffers D, Booij J, van Eck-Smit BL, Wolters $\mathrm{ECh}$, Berendse HW. Idiopathic hyposmia as a preclinical sign of Parkinson's disease. Ann Neurol. 2004, 56: 173-181.

6. Mesholam RI, Moberg PJ, Mahr RN, Doty RL et al. Olfaction in neurodegenerative disease: a meta-analysis of olfactory functioning in Alzheimer's and Parkinson's diseases. Arch Neurol. 1998, 55: 84-90.

7. Huisman E, Uylings H, Hoogland PA. $100 \%$ increase of dopaminergic cells in the olfactory bulb may explain hyposmia in Parkinson's disease. Mov Disord. 2004, 19: 687-692.

8. Doty RL, LI C, Mannon LJ, Yousem DM. Olfactory dysfunction in multiple sclerosis. Relation to plaque load in inferior frontal and temporal lobes. Ann N Y Acad sci. 1998, 855: 781-786.

9. Hawkes CH, Shephard BC. Olfactory evoked responses and identification tests in neurological disease. Ann NY Acad Sci. 1998; 855: 608-615.

10. Zivadinov R, Zorzon M, Monti Bragadin L, Pagliaro G, Cazzato G. Olfactory loss in multiple sclerosis. J Neurol Sci. 1999, 168: 127-130.

11. Zorzon M, Ukmar M, Bragadin LM, et al. Olfacory dysfunction and extend of white matter abnormalities in multiple sclerosis: a clinical and MR study. Mult Scler. 2000, 6: 386-390.

12. Constantinescu CS, Raps EC, Cohen JA, West SE, Doty RL. Olfactory disturbances as the initial or most prominent symptom of multiple sclerosis. J Neurol Neurosurg Psychiatry. 1994, 57 : 1011-1012.

13. McDonald WI, Compston A, Edan G, et al. Recommended diagnostic criteria for multiple sclerosis: Guidelines from the International Panel on the Diagnosis of Multiple Sclerosis. Ann Neurol 2001, 50: 121-127.

14. Folstein MF, Folstein SE, McHugh PR. Mini-mental state. A practical method for grading the cognitive state of patients for the clinician. J Psych Res. 1975, 12: 189-198.

15. Beck AT. Depression: Causes and Treatment. Philadelphia. University of Pennsylvania Press. 2006. ISBN 0-8122-1032-1038.

16. Kurtzke JF. Rating neurologic impairment in multiple sclerosis: an expanded disability status scale (EDSS). Neurology. 1983, 33: 1444-1452.

17. Hummel T, Sekinger B, Wolf SR, Pauli E, Kobal G. Sniffin' Sticks: Olfactory performance assessed by the combined testing of odor identification, odor discrimination and ofactory threshold. Chem Senses. 1997, 22: 39-52.

18. Kobal G, Hummel T, Sekinger B, Barz S, Roscher S, Wolf S. "Sniffin'Sticks": screening of olfactory performance. Rhinology. 1996, 34: 222-226.

19. Kobal G, Klimek M, Wolfensberger $\mathrm{H}$, et al. Multicenter investigation of 1036 subjects using a standardized method for the assessment of olfactory function combining tests of odor identification, odor discrimination and olfactory thresholds. Eur Arch Ororhinolaryngol. 2000, 257: 205-211.

20. Bauknecht H-C, Jach C, Fleiner F, Sedlmaier B, Götkas Ö. 
Korrelation von objektiver Olfaktometrie und volumetrischer Messungen des Bulbus olfactorius in der MRT. RoFo 2010, 182: 163-168.

21. Strous RD, Shoenfeld Y. To smell the immune system: Olfaction, autoimmunity and brain involvement. Autoimmunity reviews. 2006, 6: 54-60.

22. Abolmaali N, Gudziol V, Hummel T. Pathology of the Olfactory Nerve. Neuroimag Clin N Am. 2008, 18: 233-242.

23. Rombaux PH, Duprez T, Hummel T. Olfactory bulb volume in the clinical assessment of olfactory dysfunction. Rhinology. 2009, 47: 3-9.

24. Goektas Ö, Fleiner F, Sedlmaier B, Bauknecht C. Correlation of olfactory dysfunction of different etiologies in MRI and comparison with subjective and objective olfactometry. Eur J of Radiology. 71, 71: 469-473.

25. Yousem DM, Geckle RJ, Bilker W, Doty RL. Olfactory bulb and tract and temporal lobe volumes. Ann N Y Acad Sci. 1998, 855: 546-555

26. Ljedo PM, Gheusi G. Olfactory processing in a changing brain. Neuroreport. 2003, 14: 1655-1663.

27. Ljedo PM, Gheusi G, Vincent JD. Information processing in the mammalian olfactory system. Physiol Rev. 2005, 85: 281-317.
28. Hummel T, Witt H, Reichmann H, Welge-Luessen A, Haehner. Immunohistochemical, volumetric and functional neuroimaging studies in patients with idiopathic Parkinson's disease. J Neurol Sci. 2010, 289:119-122.

Oender Goektas

Department of Otolaryngology Head and Neck Surgery

University of Berlin, Charité Campus Mitte

Chariteplatz 110117

Berlin

Germany

Tel: +49-30-450-655 218

Fax: +49-30-450-555 922

E-mail: oender.goektas@charite.de

ADVERTISEMENT

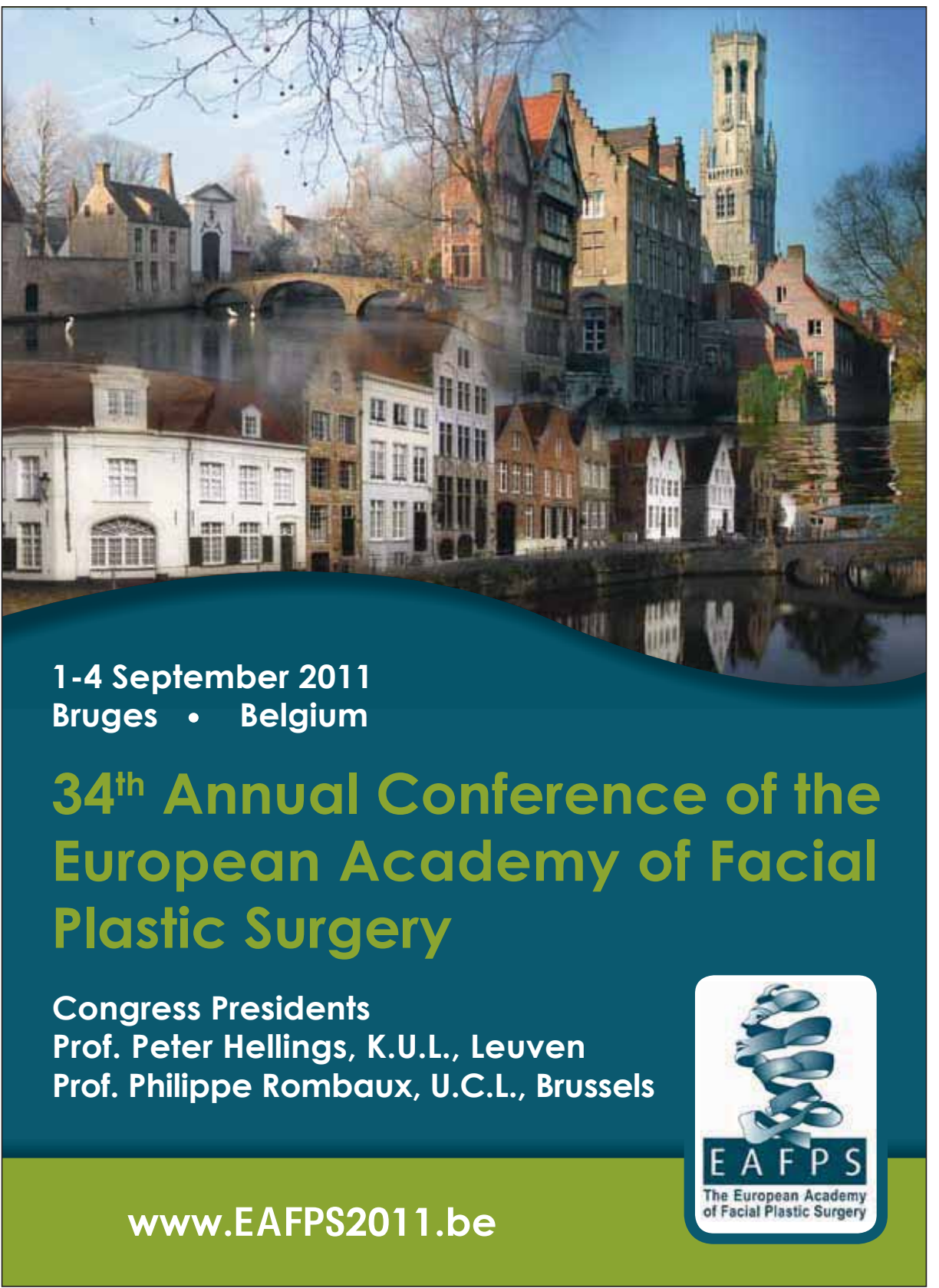

